CLINICAL STUDY

\title{
Positive association between plasma IGF1 and high-density lipoprotein cholesterol levels in adult nondiabetic subjects
}

Elena Succurro, Franco Arturi, Alessandro Grembiale, Fiorella Iorio, Irma Laino, Francesco Andreozzi, Angela Sciacqua, Marta Letizia Hribal, Francesco Perticone and Giorgio Sesti

Department of Experimental and Clinical Medicine, University Magna-Gracia of Catanzaro, Via Europa, 88100 Catanzaro, Italy

(Correspondence should be addressed to G Sesti; Email: sesti@unicz.it)

\begin{abstract}
Aims: Low IGF1 levels have been associated with an increased cardiovascular risk. It is unknown however whether IGF1 mediates the atherosclerotic process by modulating high-density lipoprotein cholesterol (HDL-C) independently from confounders. To address this issue, we evaluated the association between IGF1 levels and HDL-C in nondiabetic subjects.

Methods: A cross-sectional analysis was used in the context of the CAtanzaro MEtabolic RIsk factors Study. One thousand and four participants (aged 20-69 years), for whom HDL-C and IGF1 measurements were available, were eligible for the study.

Results: After adjusting for gender and age, IGF1 levels were positively correlated with HDL-C, and negatively correlated with body mass index (BMI), waist circumference, blood pressure (BP), triglyceride, fasting insulin, and homeostasis model assessment (HOMA). In a logistic regression model adjusted for age and gender, IGF1 in the lowest tertile $(<125 \mathrm{ng} / \mathrm{ml})$ was associated with an increased risk of having low HDL-C (odds ratio (OR) 2.14, 95\% confidence interval (CI) 1.4-3.0; $P=4 \times 10^{-5}$ ) compared with the highest tertile $(>186 \mathrm{ng} / \mathrm{ml})$. When BMI, waist circumference, total cholesterol, triglyceride, and HOMA index were added to the model, IGF1 remained significantly associated with increased risk of low HDL-C (OR 1.52, 95\% CI 1.01-2.31; P=0.04). A stepwise multivariate regression analysis in a model including age, gender, BMI, total cholesterol, triglycerides, IGF1, HOMA, and BP showed that the variables significantly associated with HDL-C were gender $(P<0.0001)$, triglycerides $(P<0.0001)$, total cholesterol $(P<0.0001)$, BMI $(P<0.0001)$, IGF1 levels $(P<0.0001)$, and HOMA $(P=0.001)$, accounting for $32.6 \%$ of its variation.

Conclusions: These data provide evidence that IGF1 may be an independent modulator for HDL-C in nondiabetic individuals.
\end{abstract}

European Journal of Endocrinology 163 75-80

\section{Introduction}

It has been suggested by several studies that low circulating levels of insulin-like growth factor 1 (IGF1) within the normal range play an important role in the development of cardiovascular diseases (1-6). Crosssectional studies have shown an inverse linear correlation between IGF1 levels and carotid intimamedia thickness in healthy individuals (1), and low circulating IGF1 levels have been associated with angiographically documented coronary artery disease (2). In subjects with acute myocardial infarction, circulating IGF1 levels on hospital admission were decreased compared with those of healthy controls, and lower levels were associated with a worse prognosis (3). Moreover, population-based prospective studies have suggested that low circulating levels of IGF1 within the normal range may predict an increased risk of ischemic heart disease $(4,5)$, and nonfatal myocardial infarction (6). In contrast with these findings, it has been reported that serum IGF1 levels are independently associated with coronary artery disease progression in young male survivors of myocardial infarction, and a reduction in serum IGF1 levels could account, at least in part, for the effect of benzafibrate on progression of focal coronary atherosclerosis (7). In addition, coronary heart disease has been reported to be independently associated with higher serum levels of IGF1 (8). Furthermore, in a crosssectional study, carried out in 6773 primary care patients, high IGF1 levels were reported to be associated with an increased risk for coronary artery disease; interestingly, in the same cohort, women, but not men, with the lower IGF1 levels also showed an increased risk for coronary artery disease (9).

The mechanism by which low IGF1 levels exert their detrimental effects on the cardiovascular system has not 
yet been completely elucidated. Low circulating IGF1 levels have been associated with several cardiovascular risk factors, including obesity (7), impaired glucose tolerance/ diabetes $(10,11)$, insulin resistance $(12,13)$, endothelial dysfunction (14), left ventricular hypertrophy (15), and inflammatory factors (16), all of which are potential contributors to the observed increased risk.

Additionally, IGF1 could mediate the atherosclerotic process by affecting high-density lipoprotein cholesterol (HDL-C), a strong, independent risk factor for cardiovascular disease $(17,18)$. HDL-C is a significant predictor of cardiovascular events even in subjects who have achieved low-density lipoprotein (LDL) cholesterol targets with statin therapy (19). Although HDL-C is one of the component of the metabolic syndrome, and low circulating IGF1 has been associated with the risk of having the metabolic syndrome (12), there is only sparse evidence in the literature on the relationship between circulating IGF1 levels and HDL cholesterol $(1,20,21)$. A positive association between serum IGF1 levels and HDL-C has been reported in 132 elderly subjects (age range, 69-91 years) (20), but not in 174 healthy individuals (age range, 18-80 years) (1), or in 81 men $(<45$ years old $)$ who survived a first myocardial infarction (7), whereas a weak negative correlation between IGF1 levels and HDL-C has been observed in 6773 primary care patients ( $>18$ years old) (9). A positive association between fetal IGF1 levels and HDL-C has been reported in 139 offspring of mothers with type 1 diabetes and 48 control subjects at birth (21). Therefore, data on the relationship between circulating IGF1 levels and HDL-C in middle-aged nondiabetic adults are limited. In particular, in a mechanistic perspective, it is poorly understood whether IGF1 has a role in modulating HDL-C independently from insulin resistance, a known modulator of HDL-C. The investigation of the putative role played by IGF 1 and insulin resistance requires the use of multivariable statistical models in large-scale studies. We used this approach with the aim to evaluate whether circulating IGF1 levels are independently associated with HDL-C in a large cohort of nondiabetic adult subjects.

\section{Methods}

The study group consisted of 1004 Caucasian subjects participating in the CAtanzaro MEtabolic RIsk factors Study (CATAMERIS), a disease prevention campaign having, at least, one cardiometabolic risk factor including age, blood pressure (BP), lipids, glucose tolerance, body mass index (BMI), and family history for diabetes $(12,16)$. Recruitment mechanisms included word-of-mouth, flyers, and newspaper advertisements. Subjects, aged 20-69 years, were excluded if they had diabetes mellitus (fasting plasma glucose $>126 \mathrm{mg} / \mathrm{dl}$ ), serum triglycerides $>400 \mathrm{mg} / \mathrm{dl}$, chronic gastrointestinal diseases associated with malabsorption, chronic pancreatitis, history of any malignant disease, history of alcohol or drug abuse, liver or kidney failure, and treatments with lipidlowering agents. After 12-h fasting, all subjects underwent anthropometrical evaluation, and a venous blood sample was drawn for laboratory determinations. Height and weight were measured to the nearest $0.5 \mathrm{~cm}$ and $0.1 \mathrm{~kg}$ respectively. BMI was calculated as body weight (kilograms) divided by the square of height (meters). Waist (at the midpoint between the lateral iliac crest and lowest rib) was measured to the nearest $0.5 \mathrm{~cm}$. Readings of clinical BP were obtained in the left arm of the supine patients, after $5 \mathrm{~min}$ of quiet rest, with a mercury sphygmomanometer. Values were calculated as the average of the last two of three consecutive measurements obtained at 3-min intervals. Glucose, triglyceride, and total and HDL cholesterol concentrations were measured by an enzymatic method (Roche). Plasma insulin concentration was determined by a chemiluminescence-based assay (Immulite, Siemens, Italy). Plasma IGF1 concentrations were determined by chemiluminescent immunoassay (Nichols Institute Diagnostic, San Juan Capistrano, CA, USA). The minimum detectable concentration was $0.03 \mathrm{mg} / \mathrm{l}$, and the maximal intra- and inter-assay coefficients of variation were 6 and $7 \%$ respectively. All samples were assayed in triplicate, and in random order, blinded to HDL values. Insulin resistance was estimated by the homeostasis model assessment (HOMA) index.

Low HDL-C was defined as levels of $40 \mathrm{mg} / \mathrm{dl}$ for men or $<50 \mathrm{mg} / \mathrm{dl}$ for women, according to the criteria of the National Cholesterol Education Program (NCEP) Adult Treatment Panel III. The study was approved by Institutional Ethics Committees, and written consent was obtained from all participants.

\section{Statistical analysis}

Triglyceride and IGF1 were natural log transformed for statistical analysis due to their skewed distribution. Partial correlation coefficients adjusted for age and gender were computed between variables. Relationships between variables were sought by multivariable linear regression analysis in order to assess their independent contribution to HDL-C. A multivariable logistic regression analysis was used to determine the association between the tertiles of IGF1 and the low HDL-C. A two-tailed $P$ value $<0.05$ was considered statistically significant. All analysis were performed using SPSS (Chicago, IL, USA) software programme Version 12.0 for Windows.

Apriori power calculation using the Russ Lenth's power analysis software, http://www.stat.uiowa.edu/ $\sim$ rlenth/Power/index.html, indicated that the sample used in this study would allow the detection of an association between HDL levels and up to ten variables with $98 \%$ power $(\alpha<0.05)$ in a regression analysis. Using the PS power analysis software, http://biostat.mc. vanderbilt.edu/PowerSampleSize, we estimated to have 
Table 1 Anthropometric and biochemical characteristics of the study subjects, and correlation. Data are means \pm S.D.

\begin{tabular}{|c|c|c|c|c|c|}
\hline & \multirow[b]{2}{*}{ Study subjects } & \multicolumn{2}{|c|}{$\begin{array}{l}\text { Age- and gender-adjusted } \\
\text { correlations between plasma IGF1 } \\
\text { levels and cardiometabolic variables }\end{array}$} & \multicolumn{2}{|c|}{$\begin{array}{l}\text { Age- and gender-adjusted } \\
\text { correlations between HDL levels } \\
\text { and cardiometabolic variables }\end{array}$} \\
\hline & & $\begin{array}{l}\text { Pearson's correlation } \\
\text { coefficient }(r)\end{array}$ & $P$ & $\begin{array}{l}\text { Pearson's correlation } \\
\text { coefficient }(r)\end{array}$ & $P$ \\
\hline Gender (M/F) & $455 / 549$ & - & - & - & - \\
\hline Age (years) & $46 \pm 11$ & $-0.41^{a}$ & $<0.0001$ & $-0.05^{\mathrm{a}}$ & 0.11 \\
\hline BMI $\left(\mathrm{kg} / \mathrm{m}^{2}\right)$ & $29.8 \pm 6.1$ & -0.22 & $<0.0001$ & -0.29 & $<0.0001$ \\
\hline Waist circumference $(\mathrm{cm})$ & $98 \pm 14$ & -0.23 & $<0.0001$ & -0.29 & $<0.0001$ \\
\hline $\mathrm{SBP}(\mathrm{mmHg})$ & $133 \pm 17$ & -0.14 & $<0.0001$ & -0.05 & 0.12 \\
\hline $\mathrm{DBP}(\mathrm{mmHg})$ & $83 \pm 10$ & -0.09 & 0.005 & -0.06 & 0.08 \\
\hline Total cholesterol (mg/dl) & $202 \pm 39$ & 0.03 & 0.22 & 0.14 & $<0.0001$ \\
\hline HDL cholesterol (mg/dl) & $52 \pm 14$ & 0.17 & $<0.0001$ & - & - \\
\hline Triglycerides $(\mathrm{mg} / \mathrm{dl})$ & $119 \pm 61$ & -0.08 & 0.007 & -0.33 & $<0.0001$ \\
\hline Fasting glucose (mgl/dl) & $92 \pm 11$ & -0.03 & 0.22 & -0.10 & 0.001 \\
\hline Fasting insulin $(\mu \mathrm{U} / \mathrm{ml})$ & $12 \pm 8$ & -0.11 & $<0.0001$ & -0.23 & $<0.0001$ \\
\hline IGF1 (ng/ml) & $168 \pm 79$ & - & - & 0.18 & $<0.0001$ \\
\hline HOMA & $3.0 \pm 2.2$ & -0.14 & $<0.0001$ & -0.24 & $<0.0001$ \\
\hline
\end{tabular}

Fasting triglyceride, insulin, and IGF1 levels were log transformed for statistical analysis, but values in the table represent a back transformation to the original scale. M, male; F, female; SBP, systolic blood pressure; DBP, diastolic blood pressure.

${ }^{a}$ Adjusted for gender.

a $97 \%$ power $(\alpha<0.05)$ to detect an association with an OR as small as 1.50 between low HDL levels and IGF1 concentrations.

\section{Results}

Anthropometric and biochemical characteristics of the study subjects are shown in Table 1. After adjusting for gender and age, IGF1 levels were positively correlated with HDL-C, and negatively correlated with BMI, waist circumference, systolic and diastolic BP, triglyceride, fasting insulin, and HOMA index (Table 1). After adjusting for gender and age, HDL-C was negatively correlated with BMI, waist circumference, triglyceride, fasting glucose, fasting insulin, and HOMA index, and positively correlated with total cholesterol (Table 1).

The prevalence of low HDL-C was $32.6 \%$ (179 out of 549 ) in women, and $28.6 \%$ (130 out of 455 ) in men. In a logistic regression model adjusted for age and gender, IGF1 in the lowest tertile $(<125 \mathrm{ng} / \mathrm{ml})$ was associated with an increased risk of having low HDL-C (odds ratio (OR) 2.14, 95\% confidence interval (CI) 1.4-3.0; $\left.P=4 \times 10^{-5}\right)$ compared with the highest tertile (>186 ng/ml). When BMI, waist circumference, total cholesterol, triglyceride, fasting glucose, and insulin levels were added to the model, IGF1 remained significantly associated with an increased risk of low HDL-C (OR 1.54, 95\% CI 1.02-2.33; P=0.04). When fasting glucose and insulin levels were replaced by HOMA index into the logistic regression model, IGF1 remained significantly associated with an increased risk of low HDL-C (OR 1.52, 95\% CI 1.01-2.31; $P=0.04$ ).

To estimate the independent contribution of the different variables to HDL-C, we carried out a stepwise forward multivariable regression analysis in a model including age, gender, BMI, total cholesterol, triglycerides, IGF1, and HOMA index. As shown in Table 2, the variables that remained significantly associated with HDL were gender $(P<0.0001)$, triglycerides $(P<0.0001)$, total cholesterol $(P<0.0001)$, BMI $(P<0.0001)$, IGF1 levels $(P<0.0001)$, and HOMA $(P=0.001)$, accounting for $32.6 \%$ of its variation. Each unit increase in log transformed IGF1 concentrations (or $2.71 \mathrm{ng} / \mathrm{dl}$ increase in actual IGF1 concentrations) was associated with a $2.96 \mathrm{mg} / \mathrm{dl}$ increase in HDL levels. The same results were obtained when HOMA was replaced by fasting insulin and glucose levels (data not shown).

Table 2 Stepwise forward multivariate regression analysis.

\begin{tabular}{|c|c|c|c|c|c|}
\hline & Partial $r^{2}(\%)$ & Total $r^{2}(\%)$ & $\beta$ & $t$ & $\boldsymbol{P}$ \\
\hline Gender & 13.0 & 13.0 & -0.299 & -10.864 & $<0.0001$ \\
\hline Triglycerides & 8.5 & 21.5 & -0.309 & -10.150 & $<0.0001$ \\
\hline Total cholesterol & 5.4 & 26.9 & 0.241 & 8.389 & $<0.0001$ \\
\hline BMI & 3.7 & 30.6 & -0.146 & -4.956 & $<0.0001$ \\
\hline IGF1 levels & 1.2 & 31.8 & 0.104 & 3.770 & $<0.0001$ \\
\hline HOMA & 0.8 & 32.6 & -0.100 & -3.415 & 0.001 \\
\hline
\end{tabular}

Independent predictors of $\mathrm{HDL}$ after stepwise forward multivariate regression analysis in a model including gender, age, BMI, total cholesterol, triglycerides, HOMA, and IGF1 levels. 


\section{Discussion}

In this study, we report a positive association between HDL-C and plasma IGF1 levels in a relatively large cohort of adult nondiabetic subjects. This observation is consistent with two previous studies carried out in elderly subjects and offspring of mothers with type 1 diabetes at birth $(20,21)$, but not with three other studies carried out in healthy individuals (1), in men who survived a first myocardial infarction (7), and in primary care patients bearing various cardiometabolic risk factors (9). It is possible that differences in clinical characteristics and concomitant treatments for cardiometabolic risk factors may explain these divergent results. Furthermore, the findings that adult patients with GH deficiency have lower HDL-C levels than agematched controls, and that their HDL-C levels are increased by a GH replacement therapy (22) support the notion that IGF1 has a relevant role in modulating serum HDL concentration.

Importantly, we found that the positive association between HDL-C and plasma IGF1 levels was independent from known modulators of plasma HDL-C including gender, adiposity, total cholesterol, triglyceride, and glucose levels.

Mechanistically, IGF1 may influence HDL-C by modulating insulin sensitivity. In fact, clinical studies carried out in patients with type 2 diabetes or with extreme insulin resistance have shown that recombinant IGF1 administration significantly increases insulin sensitivity (23). Moreover, low IGF1 levels may contribute to insulin resistance by up-regulating hybrid insulin/IGF1 receptors whose expression is increased in insulin-resistant individuals and is negatively correlated with insulin sensitivity (24). There is compelling evidence that insulin resistance plays a pathogenic role in several steps of the HDL-C metabolism $(25,26)$. Insulin resistance leads to an increased flux of free fatty acids from adipose tissue to the liver, and an enhanced synthesis of triglyceride-rich lipoproteins. This favors the exchange of core lipids, mediated by cholesterol ester transfer protein, between triglyceride-rich lipoproteins and HDL leading to triglyceride enrichment of HDL particles. These particles are good substrates for hepatic lipase, and hydrolysis of triglycerides produces smaller particles that are cleared by the kidneys (25). In addition, insulin resistance may lead to the downregulation of the ATP-binding cassette, subfamily A, member 1 , a key molecule in lipidating newly secreted apolipoprotein A-I (ApoA-I) to produce nascent HDL particles that undergo maturation in plasma, resulting in a reduction in HDL-C levels (27). In the present study, the finding that the strength of the association between IGF1 levels and low HDL-C is attenuated, but not abolished, in the multivariable analysis when HOMA index is added to the model indicates that the effects of IGF1 on HDL-C are only partly explained by the effect of IGF1 on insulin sensitivity. An alternative mechanism by which IGF1 may modulate HDL-C levels involves IGF1-dependent regulation of hepatic scavenger receptor of class BI (SR-BI). SR-BI mediates the high affinity binding of HDL and the selective uptake of HDL-derived lipids into cells (28). It has been shown in in vitro studies with the human hepatoma cell line HepG2 that IGF1 downregulates the expression of SR-BI (29). Thus, it is possible to speculate that a reduction in circulating IGF1 levels may result in the upregulation of SR-BI leading to an increased HDL-C uptake by the liver and a decrease in circulating HDL-C concentration.

The relatively large sample size, the inclusion of both sexes, and the centralization of laboratory analysis are major strengths of this study. However, our study has some limitations that require consideration. Serum HDL-C and IGF1 were measured once. Small changes in these variables would therefore be expected if the same measurements were repeated on a separate day. However, a single nonfasting IGF1 assay has been shown to effectively characterize individual levels over at least 1 year (30). Unfortunately, measurements of ApoA1 and ApoB levels were available only for 222 subjects. In this subgroup, HDL-C was positively correlated with ApoA1 $(r=0.14 ; P=0.03)$, but not with $\mathrm{ApoB}$ or $\mathrm{ApoB} / \mathrm{ApoA} 1$ ratio, after adjusting for gender and age. Further studies with adequate power are needed to determine whether IGF1 modulates HDL-C by increasing ApoA1 levels. In addition, IGFbinding proteins (IGFBPs), such as IGFBP1 and IGFBP3, and free IGF1 levels were not measured, and this should be considered another limitation of the study. Moreover, it should be noted that to estimate insulin sensitivity, we used the HOMA index which may not be as solid and reliable as a direct measurement of insulin sensitivity by a 'gold standard' test such as the euglycemic hyperinsulinemic clamp technique. However, this is not feasible in large-scale studies, and HOMA index primarily reflects insulin sensitivity at the liver level, the main organ involved in HDL-C and IGF 1 synthesis. Thus, we believe that HOMA index is a proxy measure of insulin sensitivity that is reliable with large datasets such as the one in this study. The present study is an observational study based on outpatients seen at a referral university hospital, representing subjects at increased risk for cardiometabolic disease, and, therefore, may not be extendible to the general population. Finally, the present findings are only based on Caucasian individuals, and different results might be observed in other ethnic groups.

In conclusion, we showed a positive association between HDL-C and plasma IGF1 levels in a cohort of nondiabetic adult subjects. The positive association between HDL-C and plasma IGF1 levels was independent from known modulators of plasma HDL-C including gender, adiposity, total cholesterol, triglyceride, and glucose levels. Low IGF1 levels $(<125 \mathrm{ng} / \mathrm{ml})$ were associated with an increased risk of having low HDL-C even after adjusting for several confounding variables. 
An increase of $2.71 \mathrm{ng} / \mathrm{dl}$ in IGFI concentrations was associated with a $2.96 \mathrm{mg} / \mathrm{dl}$ increase in HDL levels, thus suggesting that variations in IGF1 concentrations may have a clinically meaningful impact on HDL levels.

\section{Declaration of interest}

The authors declare that there is no conflict of interest that could be perceived as prejudicing the impartiality of the research reported.

\section{Funding}

This work was supported by the Italian Ministry of Health (grant number RF-FSR-2007-631176) to G Sesti.

\section{References}

1 Colao A, Spiezia S, Di Somma C, Pivonello R, Marzullo P, Rota F, Musella T, Auriemma RS, De Martino MC \& Lombardi G. Circulating insulin-like growth factor-I levels are correlated with the atherosclerotic profile in healthy subjects independently of age. Journal of Endocrinological Investigation 200528 440-448.

2 Spallarossa P, Brunelli C, Minuto F, Caruso D, Battistini M, Caponnetto S \& Cordera R. Insulin-like growth factor-I and angiographically documented coronary artery disease. American Journal of Cardiology 199677 200-202.

3 Conti E, Andreotti F, Sciahbasi A, Riccardi P, Marra G, Menini E, Ghirlanda G \& Maseri A. Markedly reduced insulin-like growth factor-I in the acute phase of myocardial infarction. Journal of the American College of Cardiology 200138 26-32.

4 Juul A, Scheike T, Davidsen M, Gyllenborg J \& Jørgensen T. Low serum insulin-like growth factor I is associated with increased risk of ischemic heart disease: a population-based case-control study. Circulation 2002106 939-944.

5 Laughlin GA, Barrett-Connor E, Criqui MH \& Kritz-Silverstein D. The prospective association of serum insulin-like growth factor I (IGF-I) and IGF-binding protein-1 levels with all cause and cardiovascular disease mortality in older adults: the Rancho Bernardo Study. Journal of Clinical Endocrinology and Metabolism 200489 114-120.

6 Kaplan RC, McGinn AP, Pollak MN, Kuller LH, Strickler HD, Rohan TE, Cappola AR, Xue XN \& Psaty BM. Association of total insulin-like growth factor-I, insulin-like growth factor binding protein-1 (IGFBP-1), and IGFBP-3 levels with incident coronary events and ischemic stroke. Journal of Clinical Endocrinology and Metabolism 200792 1319-1325.

7 Ruotolo G, Båvenholm P, Brismar K, Eféndic S, Ericsson CG, de Faire U, Nilsson J \& Hamsten A. Serum insulin-like growth factor-I level is independently associated with coronary artery disease progression in young male survivors of myocardial infarction: beneficial effects of bezafibrate treatment. Journal of the American College of Cardiology 200035 647-654.

8 Fischer F, Schulte H, Mohan S, Tataru MC, Köhler E, Assmann G \& von Eckardstein A. Associations of insulin-like growth factors, insulin-like growth factor binding proteins and acid-labile subunit with coronary heart disease. Clinical Endocrinology 200461 595-602.

9 Schneider HJ, Klotsche J, Saller B, Böhler S, Sievers C, Pittrow D, Ruf G, März W, Erwa W, Zeiher AM, Silber S, Lehnert H, Wittchen HU \& Stalla GK. Associations of age-dependent IGF-I SDS with cardiovascular diseases and risk conditions: crosssectional study in 6773 primary care patients. European Journal of Endocrinology $2008 \mathbf{1 5 8} 153-161$.

10 Marini MA, Succurro E, Frontoni S, Hribal ML, Andreozzi F, Lauro R, Perticone F \& Sesti G. Metabolically healthy but obese women have an intermediate cardiovascular risk profile between healthy non-obese women and obese insulin resistant women. Diabetes Care 200730 2145-2147.

11 Sandhu MS, Heald AH, Gibson JM, Cruickshank JK, Dunger DB \& Wareham NJ. Circulating concentrations of insulin-like growth factor-I and development of glucose intolerance: a prospective observational study. Lancet 2002359 1740-1745.

12 Sesti G, Sciacqua A, Cardellini M, Marini MA, Maio R, Vatrano M, Succurro E, Lauro R, Federici M \& Perticone F. Plasma concentration of insulin-like growth factor-I is independently associated with insulin sensitivity in subjects with different degree of glucose tolerance. Diabetes Care 200528 132-137.

13 Succurro E, Andreozzi F, Marini MA, Lauro R, Hribal ML, Perticone F \& Sesti G. Low plasma insulin-like growth factor-1 levels are associated with reduced insulin sensitivity and increased insulin secretion in nondiabetic subjects. Nutrition, Metabolism, and Cardiovascular Diseases 200919 713-719.

14 Perticone F, Sciacqua A, Perticone M, Laino I, Miceli S, Care' I, Galiano Leone G, Andreozzi F, Maio R \& Sesti G. Low plasma insulin-like growth factor-1 levels are associated with impaired endothelium-dependent vasodilatation in a cohort of untreated hypertensive Caucasian subjects. Journal of Clinical Endocrinology and Metabolism 200893 2806-2810.

15 Sesti G, Sciacqua A, Scozzafava A, Vatrano M, Angotti E, Ruberto C, Santillo E, Parlato G \& Perticone F. Effects of growth hormone and insulin-like growth factor- 1 on cardiac hypertrophy of hypertensive patients. Journal of Hypertension 200725 471-477.

16 Succurro E, Andreozzi F, Sciaqua A, Hribal ML, Perticone F \& Sesti G. Reciprocal association of plasma insulin-like growth factor-1 and interleukin-6 levels with cardio-metabolic risk factors in nondiabetic subjects. Diabetes Care 200831 1886-1888.

17 Gordon DJ, Probstfield JL, Garrison RJ, Neaton JD, Castelli WP, Knoke JD, Jacobs DR Jr, Bangdiwala S \& Tyroler HA. High-density lipoprotein cholesterol and cardiovascular disease: four prospective American studies. Circulation 198979 8-15.

18 Norata GD, Pirillo A \& Catapano AL. Modified HDL: biological and physiopathological consequences. Nutrition, Metabolism, and Cardiovascular Diseases 200616 371-386.

19 Barter P, Gotto AM, LaRosa JC, Maroni J, Szarek M, Grundy SM, Kastelein JJ, Bittner V, Fruchart JC \& Treating to New Targets Investigators . HDL cholesterol, very low levels of LDL cholesterol, and cardiovascular events. New England Journal of Medicine 2007 357 1301-1310.

20 Ceda GP, Dall'Aglio E, Magnacavallo A, Vargas N, Fontana V, Maggio M, Valenti G, Lee PD, Hintz RL \& Hoffman AR. The insulin-like growth factor axis and plasma lipid levels in the elderly. Journal of Clinical Endocrinology and Metabolism $1998 \mathbf{8 3}$ 499-502.

21 Nelson SM, Freeman DJ, Sattar N, Johnstone FD \& Lindsay RS. IGF-1 and leptin associate with fetal HDL cholesterol at birth: examination in offspring of mothers with type 1 diabetes. Diabetes $2007562705-2709$.

22 Götherström G, Bengtsson BA, Bosaeus I, Johannsson G \& Svensson J. A 10-year, prospective study of the metabolic effects of growth hormone replacement in adults. Journal of Clinical Endocrinology and Metabolism 200792 1442-1445.

23 Moses AC, Young SC, Morrow LA, O'Brien M \& Clemmons DR. Recombinant human insulin-like growth factor I increases insulin sensitivity and improves glycemic control in type II diabetes. Diabetes 199645 91-100.

24 Federici M, Porzio O, Lauro D, Borboni P, Giovannone B, Zucaro L, Hribal ML \& Sesti G. Increased abundance of insulin/insulin-like growth factor-I hybrid receptors in skeletal muscle of obese subjects is correlated with in vivo insulin sensitivity. Journal of Clinical Endocrinology and Metabolism 199883 2911-2915.

25 Taskinen MR. Diabetic dyslipidaemia: from basic research to clinical practice. Diabetologia 200346 733-749.

26 Bonora E, Capaldo B, Perin PC, Del Prato S, De Mattia G, Frittitta L, Frontoni S, Leonetti F, Luzi L, Marchesini G, Marini MA, Natali A, Paolisso G, Piatti PM, Pujia A, Solini A, Vettor R, Bonadonna RC \& Group of Italian Scientists of Insulin Resistance (GISIR). 
Hyperinsulinemia and insulin resistance are independently associated with plasma lipids, uric acid and blood pressure in non-diabetic subjects. The GISIR database. Nutrition, Metabolism, and Cardiovascular Diseases $2008 \mathbf{1 8}$ 624-631.

27 Timmins JM, Lee JY, Boudyguina E, Kluckman KD, Brunham LR, Mulya A, Gebre AK, Coutinho JM, Colvin PL, Smith TL, Hayden MR, Maeda N \& Parks JS. Targeted inactivation of hepatic Abca1 causes profound hypoalphalipoproteinemia and kidney hypercatabolism of apoA-I. Journal of Clinical Investigation 2005 115 1333-1342.

28 Acton S, Rigotti A, Landschulz KT, Xu S, Hobbs HH \& Krieger M. Identification of scavenger receptor SR-BI as a high density lipoprotein receptor. Science 1996271 518-520.
29 Cao WM, Murao K, Imachi H, Yu X, Dobashi H, Yoshida K, Muraoka T, Kotsuna N, Nagao S, Wong NC \& Ishida T. Insulin-like growth factor-1 regulation of hepatic scavenger receptor class BI. Endocrinology 2004145 5540-5547.

30 Kaaks R, Toniolo P, Akhmedkhanov A, Lukanova A, Biessy C, Dechaud H, Rinaldi S, Zeleniuch-Jacquotte A, Shore RE \& Riboli E. Serum C-peptide, insulin-like growth factor (IGF)-I, IGF-binding proteins, and colorectal cancer risk in women. Journal of the National Cancer Institute 200092 1592-1600.

Received 26 March 2010

Accepted 30 March 2010 